\title{
ARTICLE
}

Epidemiology and Population Health

\section{Cross-sectional associations between central and general adiposity with albuminuria: observations from 400,000 people in UK Biobank}

\author{
Pengfei Zhu ${ }^{1,2} \cdot$ Sarah Lewington $\mathbb{1}^{2} \cdot$ Richard Haynes ${ }^{1,2,3} \cdot$ Jonathan Emberson ${ }^{1,2} \cdot$ Martin J. Landray ${ }^{1,2} \cdot$ \\ David Cherney $^{4,5} \cdot$ Mark Woodward $^{6,7} \cdot$ Colin Baigent $^{1,2} \cdot$ William G. Herrington $\mathbb{D}^{1,2,3} \cdot$ Natalie Staplin ${ }^{1,2}$
}

Received: 19 December 2019 / Revised: 28 June 2020 / Accepted: 6 July 2020 / Published online: 16 July 2020

(c) The Author(s) 2020. This article is published with open access

\begin{abstract}
Background Whether measures of central adiposity are more or less strongly associated with risk of albuminuria than body mass index (BMI), and by how much diabetes/levels of glycosylated haemoglobin (HbAlc) explain or modify these associations, is uncertain.

Methods Ordinal logistic regression was used to estimate associations between values of central adiposity (waist-to-hip ratio) and, separately, general adiposity (BMI) with categories of urinary albumin-to-creatinine ratio (uACR) in 408,527 UK Biobank participants. Separate central and general adiposity-based models were initially adjusted for potential confounders and measurement error, then sequentially, models were mutually adjusted (e.g. waist-to-hip ratio adjusted for BMI, and vice versa), and finally they were adjusted for potential mediators.

Results Levels of albuminuria were generally low: 20,425 (5\%) had a uACR $\geq 3 \mathrm{mg} / \mathrm{mmol}$. After adjustment for confounders and measurement error, each 0.06 higher waist-to-hip ratio was associated with a 55\% (95\%CI 53-57\%) increase in the odds of being in a higher uACR category. After adjustment for baseline BMI, this association was reduced to 32\% (30-34\%). Each $5 \mathrm{~kg} / \mathrm{m}^{2}$ higher BMI was associated with a 47\% (46-49\%) increase in the odds of being in a higher uACR category. Adjustment for baseline waist-to-hip ratio reduced this association to $35 \%(33-37 \%)$. Those with higher HbA1c were at progressively higher odds of albuminuria, but positive associations between both waist-to-hip ratio and BMI were apparent irrespective of HbA1c. Altogether, about $40 \%$ of central adiposity associations appeared to be mediated by diabetes, vascular disease and blood pressure.

Conclusions Conventional epidemiological approaches suggest that higher waist-to-hip ratio and BMI are independently positively associated with albuminuria. Adiposity-albuminuria associations appear strong among people with normal $\mathrm{HbA1c}$, as well as people with pre-diabetes or diabetes.
\end{abstract}

These authors contributed equally: William G. Herrington, Natalie Staplin

Supplementary information The online version of this article (https:// doi.org/10.1038/s41366-020-0642-3) contains supplementary material, which is available to authorized users.

William G. Herrington

will.herrington@ndph.ox.ac.uk

1 Nuffield Department of Population Health (NDPH), Medical Research Council Population Health Research Unit at the University of Oxford, Oxford, UK

2 Clinical Trial Service Unit and Epidemiological Studies Unit, NDPH, University of Oxford, Oxford, UK

3 Oxford Kidney Unit, Churchill Hospital, Headington, Oxford, UK

\section{Background}

The rising levels of adiposity in many regions in the world may partly explain the global $\sim 30 \%$ increase in mortality directly attributed to chronic kidney disease (CKD) over the last decade [1]. Increased general adiposity, estimated from

4 Division of Nephrology, Department of Medicine, Toronto General Hospital, University of Toronto, Toronto, ON, Canada

5 Department of Physiology and Institute of Medical Sciences, and Department of Pharmacology and Toxicology, University of Toronto, Toronto, ON, Canada

6 The George Institute for Global Health, University of Sydney, Sydney, NSW, Australia

7 The George Institute for Global Health, University of Oxford, Oxford, UK 
body mass index (BMI), is associated with higher risk of advanced CKD (i.e. stage 4 or 5) [2-5]. Compared with the apparent optimum BMI (20 to $<25 \mathrm{~kg} / \mathrm{m}^{2}$ ), advanced CKD risk is increased by about one-third in those who are overweight (BMI 25 to $<30 \mathrm{~kg} / \mathrm{m}^{2}$ ), is approximately doubled in early obesity (BMI 30 to $<35 \mathrm{~kg} / \mathrm{m}^{2}$ ); and is tripled at a $\mathrm{BMI} \geq 35 \mathrm{~kg} / \mathrm{m}^{2}$ [2]. Adiposity-CKD associations may result from concomitant dysglycaemia and raised blood pressure, but the relative contribution of general and central adiposity levels to determining level of these mediators may differ. For example, studies have found that BMI is a stronger predictor of blood pressure than central adiposity $[6,7]$, whilst measures of central adiposity are a stronger predictor of insulin resistance, diabetes status [8] and risk of acute myocardial infarction $[9,10]$ than BMI. However, the large prospective studies which reported strong positive associations between BMI and advanced CKD have not reported associations of central adiposity with advanced CKD for a given BMI $[2,3,5,11]$.

Albuminuria is a marker of early CKD, predicts progression and is used for CKD staging [12]. Its use as an outcome provides an opportunity to compare the independent associations between general and central adiposity levels with risk of early kidney disease. Prior to embarking on the presented adiposity-albuminuria analyses, we performed a systematic review and identified 46 published observational studies which have examined adiposity and albuminuria (search results and study details are provided in Supplemental Tables S1, S2). Of these 46 studies, only five developed models which considered central adiposity measures following adjustment for BMI. Two smaller studies $(<5000$ participants) found no association between central adiposity and proteinuria after adjusting for BMI $[13,14]$, two other small studies (both from Chinese populations) found a positive association $[15,16]$, whilst a large $(>200,000)$ Japanese study found central adiposity to be associated with proteinuria after adjusting for BMI in men but not women [17]. Whether measures of central adiposity are more or less strongly associated with risk of albuminuria than BMI is therefore uncertain.

Whether adiposity-albuminuria associations are explained or modified by glycosylated haemoglobin (HbAlc) or dietary sodium is also uncertain. These are particularly relevant to consider following recent demonstrations of kidney protection using sodium-glucose cotransporter-2 (SGLT-2) inhibitors [18, 19], and glucagonlike peptide-1 receptor agonists $[20,21]$. These therapies reduce body weight and $\mathrm{HbA1c}$ and also seem to impact intraglomerular haemodynamics, with consequent reductions in the risk of albuminuria and progressive CKD. They are also natriuretic which is relevant, as high dietary sodium has been suggested to confound adiposity-albuminuria associations [22].
UK Biobank is a prospective cohort of 500,000 UK adults designed for exploring the causes of complex diseases in middle-to-older age [23-25]. The baseline survey included systematic recording of several different measures of central and general adiposity and measurement of urinary albumin-to-creatinine ratio (uACR), as well as $\mathrm{HbA} 1 \mathrm{c}$ and urinary sodium. Our objectives were to use the large size of UK Biobank to quantify precise associations between central and general adiposity measures with differences in the levels of albuminuria observed within an overweight general population, and to address current uncertainties.

\section{Materials and methods}

\section{Study design and participants}

During 2006-2010, 502,650 participants were recruited into UK Biobank at 22 UK-based assessment centres. Baseline assessments included a self-completed touch-screen questionnaire supplemented by interviews, standardised physical and functional measurements including bioimpedance, and the collection of biological samples. A subsample of participants has been subsequently re-surveyed. Full details of UK Biobank design have been described elsewhere [23-25].

In these analyses we excluded participants who withdrew their data $(n=133)$; and those with: self-reported cancer $(n=38,516)$, chronic obstructive pulmonary disease $(n=$ $1,563)$, or liver cirrhosis/liver failure $(n=323)$, those with a $\mathrm{BMI}<15$ or $>60 \mathrm{~kg} / \mathrm{m}^{2}(n=77)$; and those with no data on adiposity measures $(n=9,221)$, blood pressure $(n=1757)$, HbA1c $(n=30,878)$, urinary albumin $(11,649)$ or urinary creatinine $(n=6)$. A total of 408,527 participants remained, including 190,386 men and 218,141 women (Supplemental Fig. S1).

\section{Exposures}

The UK Biobank recorded four baseline measures of central adiposity: waist-to-hip ratio, waist-to-height ratio, waist circumference and percent trunk fat measured by bioimpedance [26]. Four measures of general adiposity were also recorded: BMI, height-adjusted weight, hip circumference and percent body fat. Standing height was measured using a Seca 202 telescopic height-measuring rod. Waist circumference at the level of the umbilicus and hip circumference were measured using a Wessex non-stretchable sprung tape measure. Weight, body fat (\%) and trunk fat (\%) were measured using a Tanita BC-418 MA body composition analyser. Further details are available in UK Biobank documentation [27]. Waist-to-hip ratio and BMI were selected as the primary focus of analyses due both to their widespread common use and low correlation between 
these two measures. All adiposity measures were categorised into fifths.

\section{Outcome}

Some participants had no detectable albuminuria (i.e. the concentration was below the detectable limit of $6.7 \mathrm{mg} / \mathrm{L}$ ). For those with detectable levels, uACR was calculated as a ratio of the paired albumin and creatinine measurements in the same urine sample. uACR was categorized according to the distribution of UACR or established cut-offs as follows: undetectable uACR, low "normal" (uACR 0.1 to $<1 \mathrm{mg}$ / mmol), high "normal" (uACR 1 to $<3 \mathrm{mg} / \mathrm{mmol}$ ) and albuminuria (uACR $\geq 3 \mathrm{mg} / \mathrm{mmol}$ ) [12].

\section{Statistical analysis}

In order to maximise the data and include a category for undetectable $\mathrm{UACR}$, ordinal logistic regression was used to estimate the strength of the associations between each adiposity measure and uACR. To assess the shape of the associations for each adiposity measure, participants were divided into fifths (I-V) based on the given measure. For each category of the adiposity measures, an odds ratio (OR) relative to the bottom fifth was estimated using an ordinal logistic regression model. This gives the ratio of the odds of being in a higher uACR category (i.e. low normal vs undetectable, high normal vs low normal or albuminuria vs high normal). Sensitivity analyses using any albuminuria as a binary outcome (uACR $\geq 3 \mathrm{mg} / \mathrm{mmol}$ versus undetectable or "normal" levels) were also performed.

Potential confounders and effect mediators were identified based on the assumed pathways between the exposure (adiposity) and the outcome (uACR, see model assumptions in Supplemental Fig. S2). The separate relevance of each central and general adiposity measure to uACR was then assessed in confounder-adjusted models adjusted for baseline age (continuous), ethnicity (categorical: white, mixed, Asian or Asian British, Black or Black British, Chinese, other ethnic group, don't know/missing), education (categorical: College/University degree, A levels/AS levels or equivalent, $\mathrm{O}$ levels/CSEs/NVQ/others, none of the above, prefer not to answer), Townsend deprivation index (categorical: fifths), smoking (categorical: current smoker, previous smoker, never smoker, prefer not to answer/missing), physical activity (categorical: <10MET-h/week, 10-49.9 MET-h/week, >50 MET-h/week, missing) and urinary sodium-to-creatinine ratio $\left(\mathrm{Na}^{+}: \mathrm{Cr}\right.$ ratio, an estimate of recent dietary sodium intake: fifths) [28]. Adiposity-adjusted models were then constructed by mutually adjusting for a baseline central-general adiposity pair (e.g. waist-to-hip ratio was adjusted for BMI, and vice versa) to estimate the independent effect of each central and general adiposity measure. Finally, mediator-adjusted models were further adjusted for diabetes status, duration of diabetes and any prior history of vascular disease (myocardial infarction, angina, or stroke), and systolic/diastolic blood pressure (SBP/DBP) at baseline, to assess the extent to which any effect of adiposity on UACR is through these proposed mediators. Diabetes status was categorised as diabetes (selfreported diabetes [any type] or $\mathrm{HbA} 1 \mathrm{c} \geq 6.5 \%$ ), pre-diabetes (HbA1c between 5.7 and $<6.5 \%$ ) and no diabetes $(\mathrm{HbA} 1 \mathrm{c}<$ $5.7 \%)$.

Single baseline adiposity measurements are subject to random measurement error as well as possible changes over time. Failure to account for this leads to an underestimation of the true strength of the relationship (i.e. regressiondilution bias) [29]. Other work on adiposity suggests the vast majority of regression-dilution bias for the presented exposures relates to measurement error (as regression to the mean does not increase substantially over time), and that waist and hip circumference measurements are more susceptible to measurement error than BMI [30]. To ensure comparability between different adiposity measures, the mean of repeated adiposity measurements at resurvey (about 4.3 years after the baseline survey) in 16,833 participants was used as the estimate of measurement-error adjusted adiposity levels for individuals in each baseline adiposity fifth. The results of a sensitivity analysis without any correction for potential measurement error are provided for comparison.

Since there was a generally log-linear association between adiposity and log odds of higher uACR level for the top four baseline adiposity fifths, the OR per incremental increase in adiposity value was calculated as the exponential of the slope of the inverse variance weighted regression through these four log ORs. ORs per $5 \mathrm{~kg} / \mathrm{m}^{2}$ are provided for both sexes, which equates to $1.10 \times$ the standard deviation (SD) for BMI in the study population. ORs for other adiposity measures were scaled accordingly as follows: waist-to-hip ratio per 0.06; waist-to-height ratio per 0.07 ; waist circumference per $12.1 \mathrm{~cm}$; percent trunk fat per $7.3 \%$; height-adjusted weight per $14 \mathrm{~kg}$; hip circumference per $9.2 \mathrm{~cm}$; percent body fat per $7.6 \%$ (Note: SDs are reduced after taking account of measurement error, which was corrected by multiplying the SD by the square root of the regression-dilution ratio). All analyses were performed for men and women separately with overall estimates calculated by taking an inverse variance weighted average of the sex-specific estimates. Subgroup analyses by particular characteristics were performed on the model mutually adjusted for a central/general adiposity measure at baseline (i.e. adiposity-adjusted models).

The proportional odds assumption was checked for all covariates used in the analyses, including each of the adiposity measures. For urinary sodium-to-creatinine ratio, the assumption was found to be violated. To assess whether 
urinary sodium-to-creatinine ratio was an important confounder of associations, the ORs for adiposity in a confounder-adjusted proportional odds model (not including urinary sodium-to-creatinine ratio) were compared with those from a partial proportional odds model (with the proportional odds relaxed for urinary sodium-to-creatinine), and a likelihood ratio test used to assess for any significant improvement in model fit. No important confounding effect was noted so urinary sodium-to-creatinine was omitted from models based on ordinal logistic regression. All analyses used SAS version 9.4 (SAS Institute, Cary NY, USA) and $\mathrm{R}$ version 3.5.1.

\section{Results}

\section{Baseline characteristics}

The mean age of the 408,527 included participants was 56.2 years (SD 8.1) (Table 1). Ninety-four percent ( $n=385,897)$ of participants were white, and nearly one-third $(n=$ $133,155)$ had obtained a college or university degree. Nine percent of the participants had diabetes $(n=23,637)$ or prediabetes $(n=12,507)$ and $5.5 \%(n=22,318)$ had a history of vascular disease. Mean SBP was 138 (SD 19) $\mathrm{mmHg}$.

For both men and women, the lowest correlation was between waist-to-hip ratio and BMI (men, $\rho=0.60$; women, $\rho=0.46)$. Age-adjusted correlations between BMI and other three adiposity markers (waist circumference, waist-to-height ratio and trunk fat \%) were stronger (all $\rho>0.75$ ) and similar in men and women (Supplemental Fig. S3).

Mean waist-to-hip ratio and BMI were 0.87 (0.09) and $27.4(4.7) \mathrm{kg} / \mathrm{m}^{2}$, respectively, and were slightly higher in men than women (sex differences were more marked for waist-to-hip ratio [0.93 versus 0.82$]$, Table 1/Supplemental Tables S3, 4). Higher levels of age-adjusted waistto-hip ratio and BMI were also observed in men and women with lower educational qualifications, from more deprived areas, with lower levels of physical activity, who ever smoked, and if they reported diabetes or vascular disease (Supplemental Table S5).

Two-thirds of men $(n=123,986)$ and nearly three-quarter of women $(n=157,894)$ had no detectable urinary albumin. Among those with detectable uACR, median uACR was 1.0 (IQR $0.6,2.0) \mathrm{mg} / \mathrm{mmol}$. The crude prevalence of albuminuria (uACR $\geq 3 \mathrm{mg} / \mathrm{mmol}$ ) was $5.4 \%(n=10,233)$ in men and $4.7 \%(n=10,192)$ in women (Table 1$)$.

\section{Waist-to-hip ratio}

Within-person variability for waist-to-hip ratio was higher than for BMI (Supplemental Table S6). After adjustment for confounders and measurement error, the association between waist-to-hip ratio and uACR was "J"-shaped, but log-linear if the lowest category of waist-to-hip ratio was excluded (Fig. 1). For men and women respectively, each 0.06 higher waist-to-hip ratio was associated with a $75 \%$ $(71-79 \%)$ and $40 \%(38-43 \%)$ increase in odds of higher uACR. For both sexes combined, each 0.06 higher waist-tohip ratio was associated with a 55\% (53-57\%) increase in odds. This association attenuated after adjustment for baseline BMI to a $32 \%(30-34 \%)$ increase in odds. The odds of higher uACR level reduced to $24 \%$ (22-26\%) after further adjustment for diabetes status, duration of diabetes, vascular disease and blood pressure, with model chi square values reduced from 1,297 to 770 (Supplemental Fig. S4). These results were similar when albuminuria was used as a binary outcome (Supplemental Fig. S5).

\section{Body mass index}

Like waist-to-hip ratio, the confounder-adjusted model found associations between BMI and UACR which were "J"-shaped, but were log-linear if the lowest category was excluded (Fig. 1). For men and women, a $5 \mathrm{~kg} / \mathrm{m}^{2}$ higher BMI was associated with $71 \%(68-74 \%)$ and $35 \%$ (33-37\%) increased odds of higher uACR, respectively (Fig. 1). For both sexes combined, each $5 \mathrm{~kg} / \mathrm{m}^{2}$ higher BMI was associated with a 47\% (46-49\%) increased odds of a higher uACR level. This association attenuated to a $35 \%$ (33-37\%) increased odds after adjustment for baseline waist-to-hip ratio. After further adjustment for mediators, each $5 \mathrm{~kg} / \mathrm{m}^{2}$ higher BMI adjusted for waist-to-hip ratio was associated with a $23 \%(22-25 \%)$ increase in odds of higher uACR, with model chi square values reduced from 2,731 to 1,278 (Supplemental Fig. S4).

Analogous analyses for other adiposity exposures in adiposity-adjusted models (i.e. models assessing a central adiposity measure adjusted for a general adiposity measure, or vice versa) showed a broadly similar pattern, with the exception of waist circumference associations, which increased after adjustment for hip circumference, and hip circumference associations which were not associated with albuminuria once models had adjusted for waist circumference (Supplemental Tables S7a, b and Supplemental Fig. S6). Supplemental Fig. S7 provides sensitivity analyses without adjustment for measurement error.

\section{Effects by diabetes status}

Since the presence of albuminuria in a person with diabetes may reflect a different disease process to a person without diabetes, we assessed in more detail the shape of sexspecific associations by diabetes status. Figure 2 demonstrates that diabetes status increased the odds of being in a 
Table 1 Baseline characteristics of UK Biobank, overall and by sex.

\begin{tabular}{|c|c|c|c|}
\hline Characteristics & Men $(n=190,386)$ & Women $(n=218,141)$ & All $(n=408,527)$ \\
\hline \multicolumn{4}{|l|}{ Exposure } \\
\hline \multicolumn{4}{|l|}{ Adiposity } \\
\hline Waist-to-hip ratio & $0.93(0.06)$ & $0.82(0.07)$ & $0.87(0.09)$ \\
\hline Waist-to-height ratio & $0.55(0.06)$ & $0.52(0.08)$ & $0.53(0.07)$ \\
\hline Waist circumference $(\mathrm{cm})$ & $97(11)$ & $84(12)$ & $90(13)$ \\
\hline Trunk fat $(\%)$ & $27.6(6.6)$ & $34.0(7.8)$ & $31.0(7.9)$ \\
\hline BMI $\left(\mathrm{kg} / \mathrm{m}^{2}\right)$ & $27.8(4.2)$ & $27.0(5.1)$ & $27.4(4.7)$ \\
\hline Height-adjusted weight $(\mathrm{kg})$ & $85.9(12.9)$ & $71.2(13.3)$ & $78.0(15.0)$ \\
\hline Hip circumference $(\mathrm{cm})$ & $103(8)$ & $103(10)$ & $103(9)$ \\
\hline Body fat $(\%)$ & $25.2(5.8)$ & $36.5(6.9)$ & $31.2(8.5)$ \\
\hline \multicolumn{4}{|l|}{ Confounders } \\
\hline \multicolumn{4}{|l|}{ Socio-demographics } \\
\hline Age, years & $56.4(8.2)$ & $56.1(8.0)$ & $56.2(8.1)$ \\
\hline White & $179,611(94.3 \%)$ & $206,286(94.6 \%)$ & $385,897(94.5 \%)$ \\
\hline College or University degree & $64,727(34.0 \%)$ & $68,428(31.4 \%)$ & $133,155(32.6 \%)$ \\
\hline Townsend deprivation score & $-2.2(-3.7,0.5)$ & $-2.2(-3.7,0.4)$ & $-2.2(-3.7,0.5)$ \\
\hline \multicolumn{4}{|l|}{ Lifestyle } \\
\hline Current smoker & $23,554(12.4 \%)$ & $19,084(8.7 \%)$ & $42,638(10.4 \%)$ \\
\hline Daily drinker & $48,044(25.2 \%)$ & $35,225(16.1 \%)$ & $83,269(20.4 \%)$ \\
\hline Physical activity, MET-h/week & $34.5(36.3)$ & $30.1(30.2)$ & $32.2(33.3)$ \\
\hline Urinary sodium-to-creatinine ratio $(\mathrm{mmol} / \mathrm{mmol})^{\mathrm{a}}$ & $8.8(6.0,12.3)$ & $10.4(6.9,14.9)$ & $9.6(6.4,13.7)$ \\
\hline \multicolumn{4}{|l|}{ Mediators } \\
\hline \multicolumn{4}{|l|}{ Health status } \\
\hline Diabetes $^{\mathrm{b}}$ & $14,693(7.7 \%)$ & $8,944(4.1 \%)$ & $23,637(5.8 \%)$ \\
\hline $\mathrm{HbA} 1 \mathrm{c}(\%)$ & $6.8(6.2,7.6)$ & $6.7(6.2,7.5)$ & $6.8(6.2,7.6)$ \\
\hline Duration of diabetes, years & $4.0(1.0,10.0)$ & $3.0(0.0,8.0)$ & $4.0(0.0,9.0)$ \\
\hline Pre-diabetes $^{\mathrm{b}}$ & $6,058(3.2 \%)$ & $6,449(3.0 \%)$ & $12,507(3.1 \%)$ \\
\hline HbA1c (\%) & $6.1(6.1,6.3)$ & $6.1(6.0,6.2)$ & $6.1(6.0,6.2)$ \\
\hline No diabetes ${ }^{\mathrm{b}}$ & $169,635(89.1 \%)$ & $202,748(92.9 \%)$ & $372,383(91.2 \%)$ \\
\hline HbA1c (\%) & $5.3(5.1,5.5)$ & $5.3(5.1,5.5)$ & $5.3(5.1,5.5)$ \\
\hline Any vascular disease ${ }^{c}$ & $15,366(8.1 \%)$ & $6,952(3.2 \%)$ & $22,318(5.5 \%)$ \\
\hline Systolic blood pressure (mmHg) & $141(17)$ & $135(19)$ & $138(19)$ \\
\hline Diastolic blood pressure (mmHg) & $84(10)$ & $81(10)$ & $82(10)$ \\
\hline eGFR $\left(\mathrm{mL} / \mathrm{min} / 1.73 \mathrm{~m}^{2}\right)^{\mathrm{d}}$ & $90.9(13.1)$ & $91.4(13.2)$ & $91.2(13.2)$ \\
\hline \multicolumn{4}{|l|}{ Outcome } \\
\hline Urinary albumin-to-creatinine ratio $(\mathrm{mg} / \mathrm{mmol})^{\mathrm{a}}$ & $0.9(0.6,1.9)$ & $1.2(0.7,2.2)$ & $1.0(0.6,2.0)$ \\
\hline Undetectable albumin & $123,986(65.1 \%)$ & $157,894(72.4 \%)$ & $281,880(69.0 \%)$ \\
\hline$\geq 0.1$ to $<1$ & $35,867(18.8 \%)$ & $25,207(11.6 \%)$ & $61,074(14.9 \%)$ \\
\hline$\geq 1$ to $<3$ & $20,300(10.7 \%)$ & $24,848(11.4 \%)$ & $45,148(11.1 \%)$ \\
\hline$\geq 3$ & $10,233(5.4 \%)$ & $10,192(4.7 \%)$ & $20,425(5.0 \%)$ \\
\hline
\end{tabular}

Arithmetic mean (SD), N (\%) or median (Q1, Q3) shown, unless otherwise stated.

Exclusion criteria: participants with self-reported cancer, chronic obstructive pulmonary disease or liver failure/cirrhosis; or participants with missing values of adiposity measures, blood pressure, $\mathrm{HbA1c}$, or urinary albumin-to-creatinine ratio.

$B M I$ body mass index, $H b A l c$ glycosylated haemoglobin, eGFR estimated glomerular filtration rate

${ }^{a}$ Median (Q1, Q3) among those with detectable values.

${ }^{\mathrm{b}}$ Diabetes is defined as self-reported diabetes or $\mathrm{HbA} 1 \mathrm{c} \geq 6.5 \%$, pre-diabetes is defined as HbA1c between 5.7 and $<6.5 \%$, no diabetes is defined as $\mathrm{HbA} 1 \mathrm{c}<5.7 \%$.

${ }^{\mathrm{c}}$ Any vascular diseases include heart attack, angina, or stroke.

deGFR was calculated from the Chronic Kidney Disease Epidemiology Collaboration (CKD-EPI) creatinine equation.

higher uACR category by $2-3$-fold, with pre-diabetes status being intermediate risk between diabetes and no diabetes. There was some statistical evidence that both waist-to-hip ratio and BMI appeared to be somewhat more strongly associated with uACR in those with diabetes compared with those with pre-diabetes or without diabetes $\left(P_{\text {trend }}<0.0001\right.$ and 0.01 , respectively, Fig. 3), but positive log-linear associations between both waist-to-hip ratio and BMI (after 

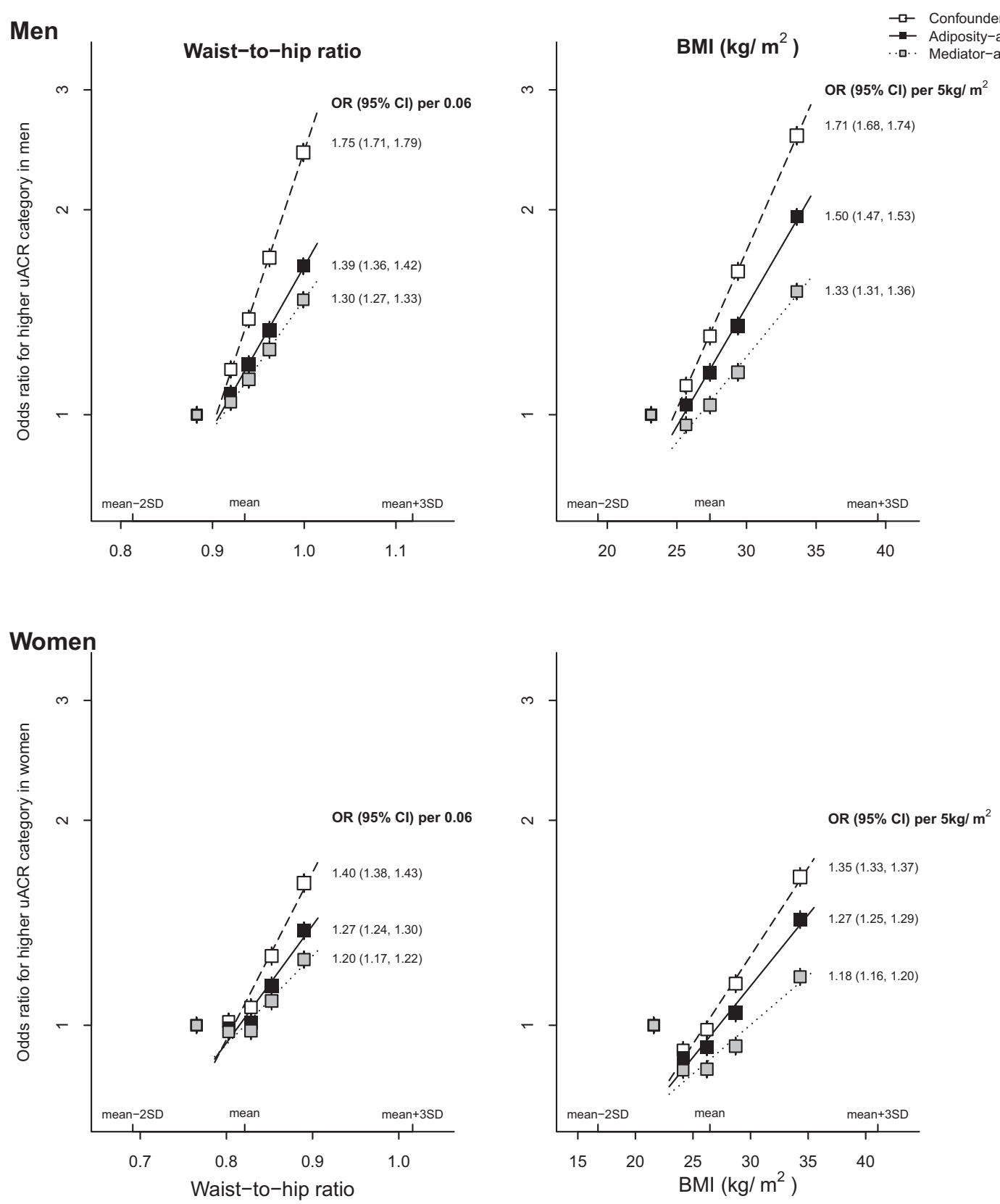

Fig. 1 Associations between adiposity (waist-to-hip ratio and BMI) and a higher urinary albumin-to-creatinine ratio category by sex. BMI body mass index, uACR urinary albumin-to-creatinine ratio, SBP systolic blood pressure, DBP diastolic blood pressure. Confounderadjusted model: adjusted for age, ethnicity, education, region, Townsend deprivation index, smoking, physical activity at baseline.

mutual adjustment) with higher uACR remained irrespective of diabetes status (Fig. 2).

\section{Effects by urinary sodium-to-creatinine ratio}

Including urinary sodium-to-creatinine ratio in models improved fit $(p<0.001)$, but any confounding effect was small, as adiposity-albuminuria associations were

Adiposity-adjusted model: further adjusted for reciprocal adiposity (i.e. waist-to-hip ratio adjusted for BMI, and BMI adjusted for waistto-hip ratio) at baseline. Mediator-adjusted model: further adjusted for diabetes status (diabetes, pre-diabetes, no diabetes), duration of diabetes, SBP, DBP and any self-reported vascular disease (heart attack, angina and stroke) at baseline.

changed little by its inclusion. The ORs per $5 \mathrm{~kg} / \mathrm{m}^{2}$ higher BMI were $1.47(1.43-1.50)$ versus 1.46 (1.43-1.50) before and after adjustment for urinary sodium-to-creatinine ratio, and per 0.06 higher waist-tohip ratio were $1.62(1.58-1.67)$ versus 1.62 (1.57-1.67), respectively. In women but not men, there was a suggestion that higher urinary sodium-to-creatinine ratio increased the odds of being in a higher uACR category 


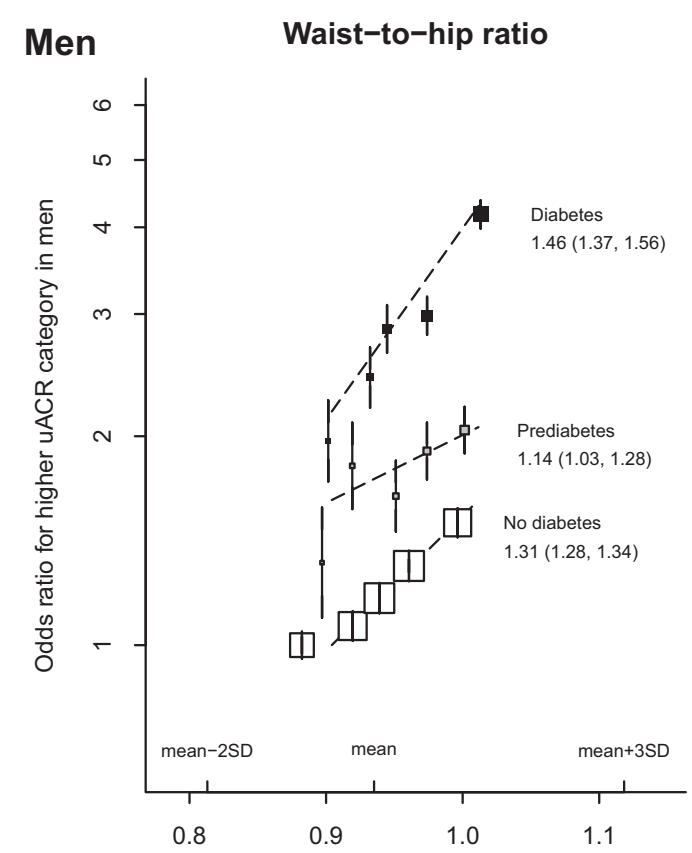

\section{BMI $\left(\mathbf{k g} / \mathrm{m}^{2}\right)$}

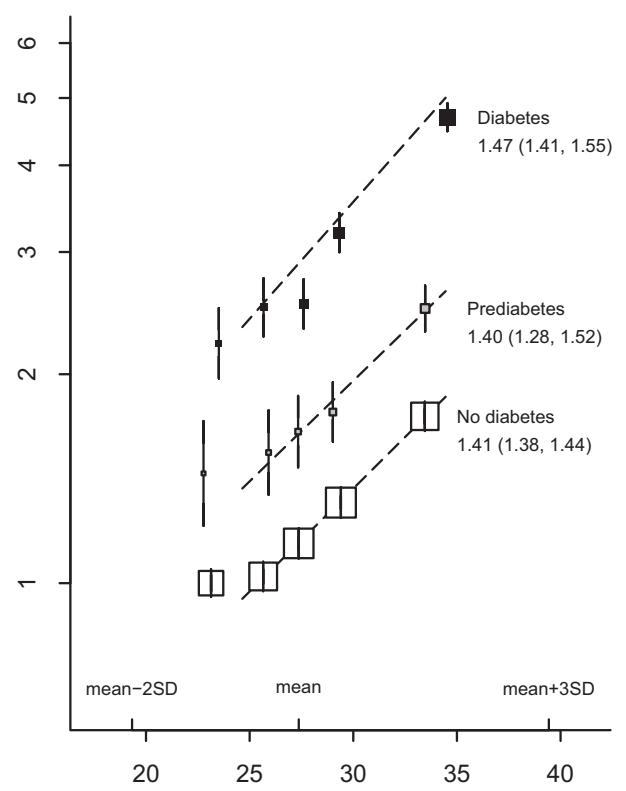

\section{Women}

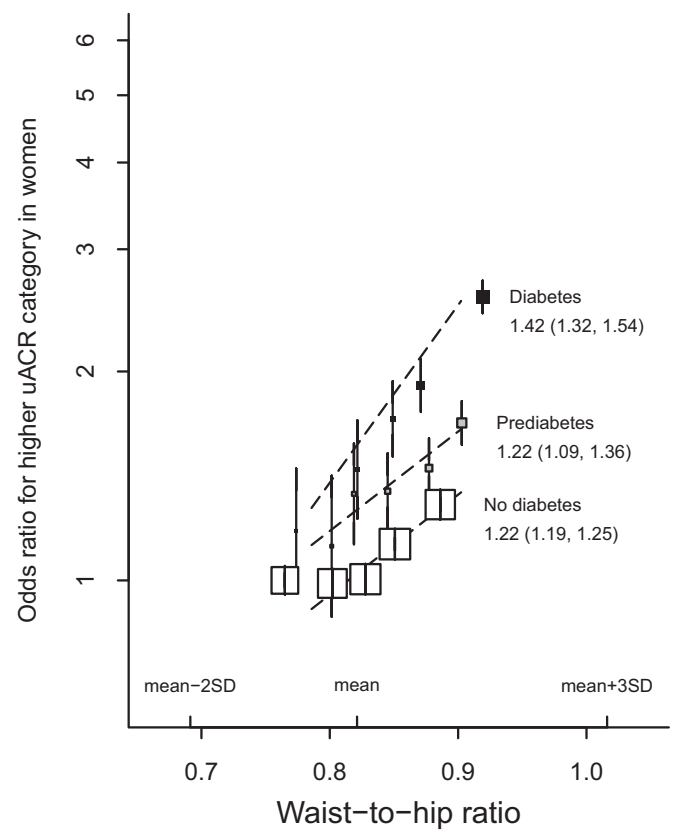

Fig. 2 Associations between adiposity (waist-to-hip adjusted for BMI and BMI adjusted for waist-to-hip ratio) and a higher urinary albumin-to-creatinine ratio category by sex and by diabetes status. BMI body mass index, uACR urinary albumin-to-

(Supplemental Fig. S8). Nevertheless, in both men and women, the shape and size of associations between waistto-hip ratio and BMI (after mutual adjustment) with higher categories of uACR appeared to be similar in those with the highest and lowest halves of urinary sodium-tocreatinine ratio.

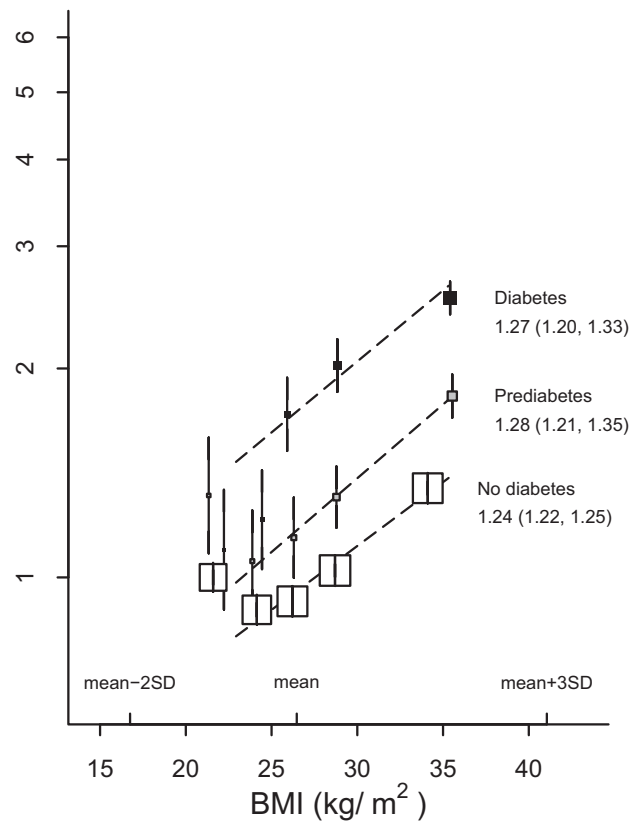

creatinine ratio. Models were adjusted for confounders (age, ethnicity, education, region, Townsend deprivation index, smoking, physical activity) and a reciprocal adiposity measurement (i.e. waist-to-hip ratio adjusted for BMI, BMI adjusted for waist-to-hip ratio).

\section{Effects by other characteristics}

Other than sex and diabetes status, the only other consistent effect modifier was age. Those who were younger appeared to have somewhat stronger associations between waist-tohip ratio and BMI (after mutual adjustment) with increased 
odds of higher levels of uACR than those who were older $\left(P_{\text {trend }}=0.0013\right.$ and 0.0017 , respectively; Fig. 3$)$.

\section{Discussion}

UK Biobank provides an opportunity to estimate precise associations between a range of different measures of adiposity and albuminuria in middle-aged population which was, on average, overweight. There were four main findings from these analyses. First, the study provides the clearest evidence about the independent relevance of central adiposity to risk of albuminuria to date: increasing levels of central adiposity is associated with higher levels of albuminuria independent of measures of general adiposity. Each standard deviation higher waist-to-hip ratio appears to be as important as each standard deviation higher BMI. Secondly, although these associations are stronger in men than women, they clearly exist in both sexes. Thirdly, dietary sodium (estimated using urinary sodium-to-creatinine ratio) does not appear to confound adiposity-albuminuria associations. Lastly, adiposity associations were clearly evident in those without diabetes as well as those with pre-diabetes and diabetes, and models which included diabetes, blood pressure and vascular disease showed that such factors altogether explained about $40 \%$ of the observed central adiposity-albuminuria associations.

The associations between both waist-to-hip ratio and BMI with higher levels of albuminuria may represent early disturbances in intraglomerular haemodynamics resulting from excess adiposity. General population data with iohexol measured glomerular filtration rate have demonstrated that central adiposity [31] and impaired fasting glucose [32] among people without diabetes are associated with measured hyperfiltration (which is considered to be a precursor to albuminuria and CKD development). Such data suggest adiposity and small changes in glycaemic control can alter intraglomerular haemodynamics and may therefore conceivably affect albuminuria measurements within the "normal range". Disturbances of tubuloglomerular feedback though upregulating tubular SGLT-2 function is one potential mechanism [33]. However, if this was the key mechanism, one would expect adiposity-albuminuria associations to be substantially weaker among those with normal HbAlc, and this does not appear to be the case (Fig. 2). Our findings extend and are consistent with other studies which have found the relative strength of the association between BMI and risk of advanced CKD risk is similar in shape and size to people with and without diabetes [2, 3, 5]. Taken together with randomised trial evidence [34], these conventional observational analyses suggest important pathways independent of glycaemic control and systemic blood pressure could be responsible for mediating some of adiposity- related renal risk. Such pathways may include altered intrarenal haemodynamics, perhaps through activation of the renin-angiotensin-aldosterone system, and potentially augmented levels of pro-inflammatory cascades [33].

In addition to metabolism-related pathways, renal sodium handling has been implicated in the pathogenesis of glomerular hyperfiltration and CKD progression [35]. A previous study using 24-h urinary collections to estimate dietary sodium and identify hyperfiltration raised a hypothesis that high dietary sodium may confound associations between adiposity and hyperfiltration [22]. The presented analyses did not confirm this hypothesis: no pathophysiological synergism between the effects of obesity [36] and high dietary sodium on albuminuria was evident with adiposity-albuminuria associations unmodified by levels of urinary sodium (Supplemental Fig. S8). These findings are consistent with results of trials of salt-restriction which have not demonstrated any clear effect of low dietary salt on proteinuria or changes in renal function [37].

The presented analyses support the need to tackle the high prevalence of overweight and obesity which is high and rising in many areas of the world $[38,39]$. In the United Kingdom, prevalence of obesity (BMI $\geq 30 \mathrm{~kg} / \mathrm{m}^{2}$ ) increased from 15 to $27 \%$ between 1993 and 2015 [40]. For those that are obese and have already developed albuminuria, low calorie diets and increased physical activity (or bariatric surgery) address the root cause and may reverse glomerular hyperfiltration and hypertension [34]. Lowering intraglomerular pressure using renin-angiotensin system inhibitors may be a useful therapeutic strategy, and SGLT-2 inhibition may have a broader range of renoprotective effects including reductions in body weight, hyperglycaemia as well as intraglomerular hypertension. Their effects on CKD progression outcomes are being assessed in large-scale CKD trials, including people without diabetes [41].

The present UK Biobank study is at least 10-times larger than any of the other reported studies of a Caucasian population to consider central adiposity and/or general measures with levels of albuminuria (Supplemental Table S2). However, there are some limitations. First, the cross-sectional and observational nature of the data limits assessment of the temporality of associations precluding assessment of causality. Secondly, measurement error in covariates in the model may lead to an underestimate of the importance of meditators (particularly blood pressure). Future analyses using genetically determined adiposity and Mendelian randomisation approaches could be used to address both these issues. Thirdly, although albuminuria is a strong predictor of advanced CKD [42] and is used to define and stage CKD in people with and without diabetes [12], it is not as important to patients as the need for renal replacement therapy. These novel findings therefore still need to be tested in prospective cohorts which are large and long enough to have recorded a 


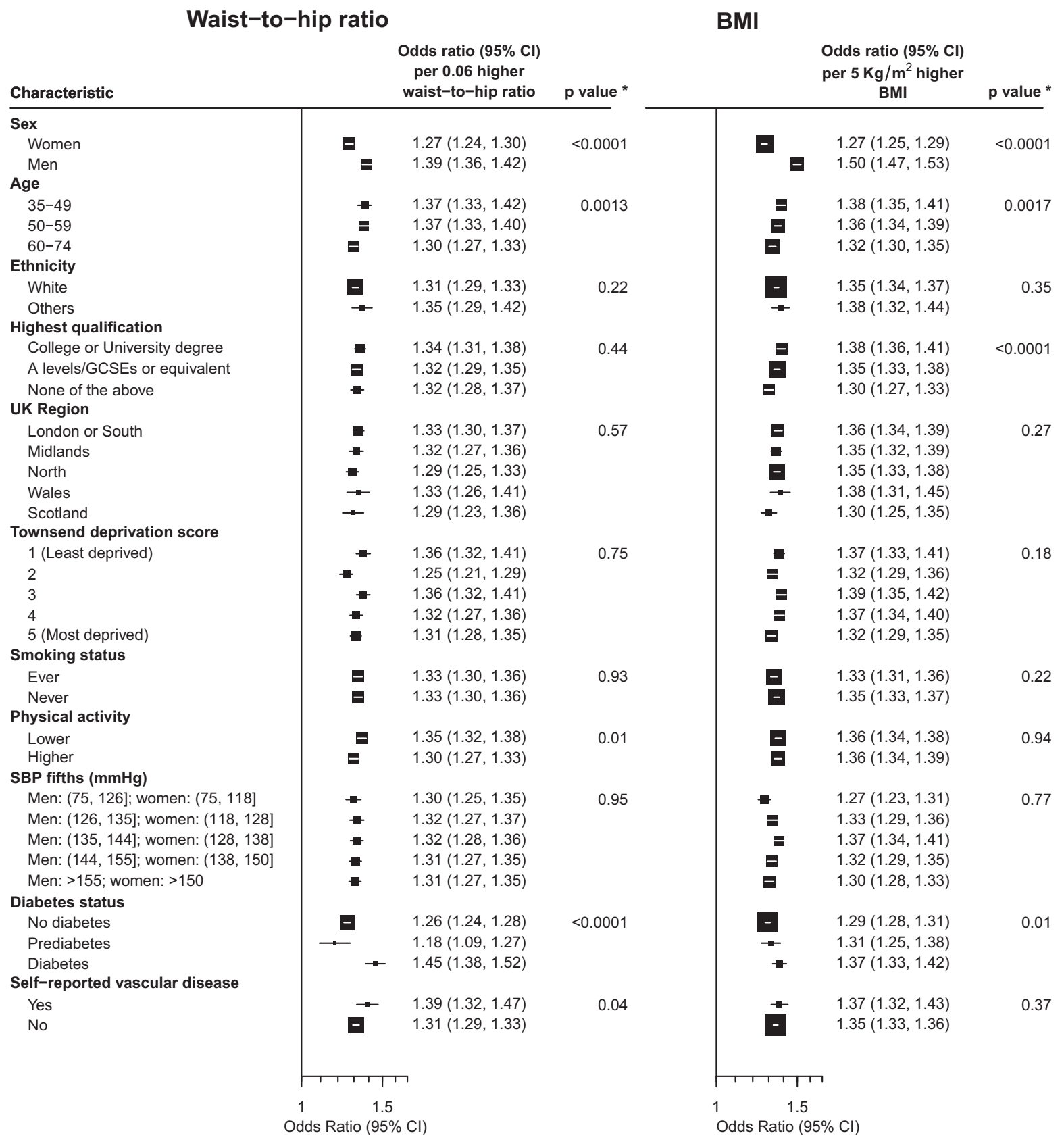

Fig. 3 Associations between incremental increase in adiposity (waist-to-hip ratio adjusted for BMI and BMI adjusted for waistto-hip ratio) and a higher urinary albumin-to-creatinine ratio category by participant characteristics. ${ }^{*} p$ value for trend or heterogeneity test. BMI body mass index, SBP systolic blood pressure. Log ORs for a higher uACR category per incremental increase in adiposity by subgroups are the inverse variance weighted averages of

sufficient number of incident end-stage kidney disease outcomes [5]. Fourthly, the use of spot samples to estimate dietary sodium intake has recognised limitations, but longer duration urine collections are less feasible in large-scale studies [43]. Lastly, UK Biobank is predominately a study of Caucasians and may not extrapolate to other populations around the world. the sex-specific log ORs by subgroups (calculated as the slopes of the inverse variance weighted regressions through the log ORs of the top four adiposity categories in each subgroup). Model was adjusted for confounders (age, ethnicity, education, region, Townsend deprivation index, smoking, physical activity where relevant) and reciprocal adiposity (i.e. waist-to-hip ratio adjusted for BMI, BMI adjusted for waist-to-hip ratio) at baseline.

In conclusion, both higher central and general adiposity are independently associated with albuminuria and appear to be similarly important. Dietary sodium levels (estimated by urinary sodium) do not appear to confound these associations, and although increasing HbAlc clearly increases risk of albuminuria, adiposity-albuminuria associations appear strong among people with normal or elevated levels of HbA1c. As diabetes, 
blood pressure and prior vascular disease appear to explain about $40 \%$ of the central adiposity-albuminuria association, genetic epidemiological approaches and experimental work should be used to assess whether other mechanisms might underpin these observed associations.

Acknowledgements This research has been conducted using the UK Biobank Resource (application number 15595). The funders had no role in the analysis or interpretation of the data and no role in the decision to submit the manuscript for publication. The Medical Research Council Population Health Research Unit at the University of Oxford, which is part of the Clinical Trial Service Unit and Epidemiological Studies Unit (CTSU), receives core funding from the United Kingdom Medical Research Council. These analyses were also supported by Health Data Research UK and the NIHR Oxford Biomedical Research Centre. WGH is supported by a Medical Research Council Kidney Research UK Professor David Kerr Clinician Scientist Award.

Author contributions WGH conceived the study. PFZ developed the study design and performed the literature search and analyses under the supervision of SL, WGH and NS. PFZ and NS had full access to the data. PFZ, NS and WGH wrote the first draft of the manuscript. MJL contributed to UK Biobank data collection. All authors contributed to data interpretation and revision of the manuscript.

\section{Compliance with ethical standards}

Conflict of interest This paper has not been published previously in whole or part. CTSU has a staff policy of not accepting honoraria or other payments from the pharmaceutic industry, expect for the reimbursement of costs to participate in scientific meetings. MW reports personal fees from Amgen and from Kirin outside the submitted work.

Publisher's note Springer Nature remains neutral with regard to jurisdictional claims in published maps and institutional affiliations.

Open Access This article is licensed under a Creative Commons Attribution 4.0 International License, which permits use, sharing, adaptation, distribution and reproduction in any medium or format, as long as you give appropriate credit to the original author(s) and the source, provide a link to the Creative Commons license, and indicate if changes were made. The images or other third party material in this article are included in the article's Creative Commons license, unless indicated otherwise in a credit line to the material. If material is not included in the article's Creative Commons license and your intended use is not permitted by statutory regulation or exceeds the permitted use, you will need to obtain permission directly from the copyright holder. To view a copy of this license, visit http://creativecommons. org/licenses/by/4.0/.

\section{References}

1. Neuen BL, Chadban SJ, Demaio AR, Johnson DW, Perkovic V. Chronic kidney disease and the global NCDs agenda. BMJ Glob Health. 2017;2:e000380 https://doi.org/10.1136/bmjgh-2017-000380

2. Herrington WG, Smith M, Bankhead C, Matsushita K, Stevens S, Holt $\mathrm{T}$, et al. Body-mass index and risk of advanced chronic kidney disease: Prospective analyses from a primary care cohort of 1.4 million adults in England. PLoS ONE. 2017;12:e0173515 https://doi.org/10.1371/journal.pone.0173515

3. Hsu CY, McCulloch CE, Iribarren C, Darbinian J, Go AS. Body Mass Index and Risk for End-Stage Renal Disease. Ann Intern
Med. 2006;144:21-28. https://doi.org/10.7326/0003-4819-144-1200601030-00006

4. Iseki K, Ikemiya Y, Kinjo K, Inoue T, Iseki C, Takishita S. Body mass index and the risk of development of end-stage renal disease in a screened cohort. Kidney Int. 2004;65:1870-6. https://doi.org/ 10.1111/j.1523-1755.2004.00582.x

5. Chang AR, Grams ME, Ballew SH, Bilo H, Correa A, Evans M, et al. Adiposity and risk of decline in glomerular filtration rate: meta-analysis of individual participant data in a global consortium. BMJ. 2019;364:k5301. https://doi.org/10.1136/bmj.k5301

6. Chen Z, Smith M, Du H, Guo Y, Clarke R, Bian Z, et al. Blood pressure in relation to general and central adiposity among 500 000 adult Chinese men and women. Int $\mathbf{J}$ Epidemiol. 2015;44:1305-19. https://doi.org/10.1093/ije/dyv012

7. Gnatiuc L, Alegre-Diaz J, Halsey J, Herrington WG, LopezCervantes M, Lewington S, et al. Adiposity and Blood Pressure in 110000 Mexican Adults. Hypertension. 2017;69:608-14. https:// doi.org/10.1161/HYPERTENSIONAHA.116.08791

8. Lee CM, Huxley RR, Wildman RP, Woodward M. Indices of abdominal obesity are better discriminators of cardiovascular risk factors than BMI: a meta-analysis. J Clin Epidemiol. 2008;61:646-53. https://doi.org/10.1016/j.jclinepi.2007.08.012

9. Yusuf S, Hawken S, Ounpuu S, Bautista L, Franzosi MG, Commerford $\mathrm{P}$, et al. Obesity and the risk of myocardial infarction in 27,000 participants from 52 countries: a case-control study. Lancet. 2005;366:1640-9. https://doi.org/10.1016/S0140-6736(05)67663-5

10. Peters SAE, Bots SH, Woodward M. Sex differences in the association between measures of general and central adiposity and the risk of myocardial infarction: results from the UK Biobank. J Am Heart Assoc. 2018;7:e008507. https://doi.org/10.1161/JAHA. 117.008507

11. Vivante A, Golan E, Tzur D, Leiba A, Tirosh A, Skorecki K, et al. Body mass index in 1.2 million adolescents and risk for end-stage renal disease. Arch Intern Med. 2012;172:1644-50. https://doi. org/10.1001/2013.jamainternmed.85

12. Kidney Disease Improving Global Outcome (KDIGO) 2012 clinical practice guideline for the evaluation and management of chronic kidney disease. 2012. www.kdigo.org. Accessed 23 May 2020.

13. Gojaseni P, Phaopha A, Chailimpamontree W, Pajareya T, Chittinandana A. Prevalence and risk factors of microalbuminuria in Thai nondiabetic hypertensive patients. Vasc Health Risk Manag. 2010;6:157-65. https://doi.org/10.2147/vhrm.s9739

14. Seo WJ, Lee GM, Hwang JH, Lee MN, Kang HC. Association between Body Mass Index, Waist Circumference and Prevalence of Microalbuminuria in Korean Adults of Age 30 Years and Older without Diabetes, Hypertension, Renal Failure, or Overt Proteinuria: The 2013 Korean National Health and Nutrition Examination Survey. Korean J Fam Med. 2016;37:57-63. https://doi. org/10.4082/kjfm.2016.37.1.57

15. Wang Z, Ding L, Huang X, Chen Y, Sun W, Lin L, et al. Abdominal adiposity contributes to adverse glycemic control and albuminuria in Chinese type 2 diabetic patients: a cross-sectional study. J Diabetes. 2017;9:285-95. https://doi.org/10.1111/1753-0407

16. Lin WY, Pi-Sunyer FX, Liu CS, Li CI, Davidson LE, Li TC, et al. Central obesity and albuminuria: both cross-sectional and longitudinal studies in Chinese. PLoS ONE. 2012;7:e47960. https:// doi.org/10.1371/journal.pone.0047960

17. Sato Y, Fujimoto S, Konta T, Iseki K, Moriyama T, Yamagata $\mathrm{K}$, et al. U-shaped association between body mass index and proteinuria in a large Japanese general population sample. Clin Exp Nephrol. 2014;18:75-86. https://doi.org/10.1007/s10157013-0809-5

18. Wanner C, Inzucchi SE, Lachin JM, Fitchett D, von Eynatten M, Mattheus M, et al. Empagliflozin and progression of kidney 
disease in type 2 diabetes. N Engl J Med. 2016;375:1801-2. https://doi.org/10.1056/NEJMc1611290

19. Perkovic V, Jardine MJ, Neal B, Bompoint S, Heerspink HJL, Charytan DM, et al. Canagliflozin and renal outcomes in type 2 diabetes and nephropathy. N Engl J Med. 2019;380:2295-30. https://doi.org/10.1056/NEJMoa1811744

20. Gerstein HC, Colhoun HM, Dagenais GR, Diaz R, Lakshmanan M, Pais P, et al. Dulaglutide and renal outcomes in type 2 diabetes: an exploratory analysis of the REWIND randomised, placebo-controlled trial. Lancet. 2019;394:131-8. https://doi.org/ 10.1016/S0140-6736(19)31150-X

21. Cherney DZI, Verma S, Parker JD. Dulaglutide and renal protection in type 2 diabetes. Lancet Diabetes Endocrinol. 2018;6:588-90. https://doi.org/10.1016/S2213-8587(18)30125-6

22. Ogna A, Forni Ogna V, Bochud M, Guessous I, Paccaud F, Burnier $\mathrm{M}$, et al. Association between obesity and glomerular hyperfiltration: the confounding effect of smoking and sodium and protein intakes. Eur J Nutr. 2016;55:1089-97. https://doi.org/10. 1007/s00394-015-0923-0

23. UK Biobank: www.ukbiobank.ac.uk. Accessed 23 May 2020.

24. UK Biobank: Protocol for a large-scale prospective epidemiological resource. 2007. https://www.ukbiobank.ac.uk/wp-content/uploads/ 2011/11/UK-Biobank-Protocol.pdf. Accessed 23 May 2020.

25. Sudlow C, Gallacher J, Allen N, Beral V, Burton P, Danesh J, et al. UK biobank: an open access resource for identifying the causes of a wide range of complex diseases of middle and old age. PLoS Med. 2015;12:e1001779. https://doi.org/10.1371/journal. pmed.1001779

26. National Obesity Observatory. Measures of central adiposity as an indicator of obesity. 2009.

27. UK Biobank. Anthropometry. 2011. http://biobank.ctsu.ox.ac.uk/ crystal/crystal/docs/Anthropometry.pdf. Accessed 23 May 2020.

28. Mente A, O’Donnell MJ, Dagenais G, Wielgosz A, Lear SA, McQueen MJ, et al. Validation and comparison of three formulae to estimate sodium and potassium excretion from a single morning fasting urine compared to $24-\mathrm{h}$ measures in 11 countries. J Hypertens. 2014;32:1005-14. https://doi.org/10.1097/ hjh.0000000000000122

29. Clarke R, Shipley M, Lewington S, Youngman L, Collins R, Marmot M, et al. Underestimation of risk associations due to regression dilution in long-term follow-up of prospective studies. Am J Epidemiol. 1999;150:341-53. https://doi.org/10.1093/ oxfordjournals.aje.a010013

30. Wormser D, White IR, Thompson SG, Wood AM. Withinperson variability in calculated risk factors: comparing the aetiological association of adiposity ratios with risk of coronary heart disease. Int J Epidemiol. 2013;42:849-59. https://doi.org/ 10.1093/ije/dyt077

31. Stefansson VT, Schei J, Jenssen TG, Melsom T, Eriksen BO. Central obesity associates with renal hyperfiltration in the nondiabetic general population: a cross-sectional study. BMC Nephrol. 2016;17:172. https://doi.org/10.1186/s12882-016-0386-4
32. Melsom T, Mathisen UD, Ingebretsen OC, Jenssen TG, Njolstad I, Solbu MD, et al. Impaired fasting glucose is associated with renal hyperfiltration in the general population. Diabetes Care. 2011;34:1546-51. https://doi.org/10.2337/dc11-0235

33. Sasson AN, Cherney DZ. Renal hyperfiltration related to diabetes mellitus and obesity in human disease. World $\mathrm{J}$ Diabetes. 2012;3:1-6. https://doi.org/10.4239/wjd.v3.i1.1

34. Look AHEAD Research Group. Effect of a long-term behavioural weight loss intervention on nephropathy in overweight or obese adults with type 2 diabetes: a secondary analysis of the Look AHEAD randomised clinical trial. The Lancet Diabetes \& Endocrinology. 2014;2:801-9. https://doi.org/10.1016/S22138587(14)70156-1

35. Lovshin JA, Cherney DZ. Sodium transport in diabetes: two sides to the coin. Nat Rev Nephrol. 2019;15:125-6. https://doi.org/10. 1038/s41581-018-0106-3

36. D'Agati VD, Chagnac A, de Vries AP, Levi M, Porrini E, Herman-Edelstein $M$, et al. Obesity-related glomerulopathy: clinical and pathologic characteristics and pathogenesis. Nat Rev Nephrol. 2016;12:453-71. https://doi.org/10.1038/nrneph.2016. 75nrneph.2016.75

37. Aburto NJ, Ziolkovska A, Hooper L, Elliott P, Cappuccio FP, Meerpohl JJ. Effect of lower sodium intake on health: systematic review and meta-analyses. BMJ. 2013;346:f1326. https://doi.org/ 10.1136/bmj.f1326

38. Ng M, Fleming T, Robinson M, Thomson B, Graetz N, Margono C, et al. Global, regional, and national prevalence of overweight and obesity in children and adults during 1980-2013: a systematic analysis for the Global Burden of Disease Study 2013. Lancet. 2014;384:766-81. https://doi.org/10.1016/S0140-6736(14)60460-8

39. Stevens GA, Singh GM, Lu Y, Danaei G, Lin JK, Finucane MM, et al. National, regional, and global trends in adult overweight and obesity prevalences. Popul Health Metr. 2012;10:22. https://doi. org/10.1186/1478-7954-10-22

40. Statistics on Obesity, Physical Activity and Diet. England; 2018. Available at www.digital.nhs.uk. Accessed 13 July 2020.

41. Herrington WG, Preiss D, Haynes R, von Eynatten M, Staplin N, Hauske SJ, et al. The potential for improving cardio-renal outcomes by sodium-glucose co-transporter- 2 inhibition in people with chronic kidney disease: a rationale for the EMPA-KIDNEY study. Clin Kidney J. 2018;11:749-61. https://doi.org/10.1093/ ckj/sfy090

42. Mafham MM, Staplin N, Emberson J, Haynes R, Herrington W, Reith C, et al. Prognostic utility of estimated albumin excretion rate in chronic kidney disease: results from the Study of Heart and Renal Protection. Nephrol Dial Transplant. 2018;33:257-64. https://doi.org/10.1093/ndt/gfw396

43. Jedrusik P, Symonides B, Gaciong Z. Estimating 24-hour urinary sodium, potassium, and creatinine excretion in hypertensive patients: can we replace 24-hour urine collection with spot urine measurements? Pol Arch Intern Med. 2019;129:506-15. https:// doi.org/10.20452/pamw.14872 